\title{
Hierarchically Structured Coatings by Colorless Polydopamine Thin Layer and Polymer Brush Layer
}

\author{
Hiroto Kohma, Kanako Uradokoro, Michinari Kohri*, Tatsuo Taniguchi and Keiki Kishikawa \\ Graduate School of Engineering, Chiba University, 1-33 Yayoi-cho, Inage-ku, Chiba 263-8522, Japan \\ * Corresponding author: e-mail: kohri@faculty.chiba-u.jp
}

\begin{abstract}
Herein, we describe a general and versatile procedure to fabricate hierarchically structured coatings onto material surfaces using colorless polydopamine (PDA) thin layer and polymer brush layer. Firstly, atom transfer radical polymerization (ATRP) initiator-containing colorless PDA layer was coated onto various types of material surfaces. Then, surface-initiated ATRP was conducted to produce functional polymer brush layer. This methodology will be applied to a broad range of materials to modification of surface properties. Furthermore, one-step hierarchically structured coatings of materials surface were also investigated.

Key words: Colorless polydopamine layer, Polymer brush layer, Hierarchically structures coatings,

Surface-initiated ATRP
\end{abstract}

\section{INTRODUCTION}

The functionalization of solid surfaces is an attractive topic in material science for their scientific significance and numerous potential applications. Surface-initiated atom transfer radical polymerization (SI-ATRP) is an extremely method for surface modification [1-6]. We have reported the preparation of functionalized polystyrene (PSt) particles with hydrophilic polymer shell layers by SI-ATRP in water [7]. ATRP initiator-bearing PSt core particles were prepared by emulsifier-free emulsion polymerization of styrene and ATRP initiator-containing monomer, i.e., 2-chloropropionyloxyethyl methacrylate. Hydrophilic monomers were then polymerized onto PSt particles. Usually, the introduction of ATRP-initiating groups onto material surfaces is carried out using individual surface modifiers; for example, thiol derivatives for gold particles [8], silane-coupling reagents [9] and phosphate-functionalized derivatives [10] for inorganic particles, and vinyl group bearing compound for polymer particles [7]. Thus, the development of versatile strategies for the introduction of ATRP initiator to materials surface is essential for technological application.

Since the emergence of surface modification techniques, including polydopamine (PDA) layers using a single deposition process that is based on the oxidative self-polymerization of dopamine hydrochloride (DA) [11], the number of studies concerning the functionalization of material surfaces via PDA coating has increased [12-16]. While PDA is simple to apply to cover onto various kinds of substrates, most of conventional PDA layers show considerable dark brown coloration, restricting their use for some practical applications such as optical materials and cosmetics. To overcome this drawback, we recently demonstrated the preparation of a colorless PDA layer containing ATRP initiator prepared by the in situ reaction of DA with 2-bromoisobutyryl bromide (BiBB); The synthesized colorless PDA layer is designated $\mathrm{PDA} / \mathrm{BiBB}_{2}([\mathrm{BiBB}] /[\mathrm{DA}]=2$, the ratio uses the molar concentrations of $\mathrm{DA}$ and $\mathrm{BiBB}$ ) [17]. $\mathrm{PDA} / \mathrm{BiBB}_{2}$ layer was placed onto PSt particles, and then the surface of obtained particles was coated with polymer brush layers via SI-ATRP [18]. It would be great interest to explore the feasibility of using $\mathrm{PDA} / \mathrm{BiBB}_{2}$ layer to various types of material surfaces instead of PSt particles.

Herein, we reported a general and versatile procedure to fabricate hierarchically structured coatings onto material surfaces using colorless PDA thin layer and polymer brush layer (Fig. 1). ATRP initiator-bearing colorless PDA layer (PDA/BiBB 2 layer) has been prepared onto various types substrates, e.g., glass plate, flexible polypropylene (PP) film, filter paper, silica $\left(\mathrm{SiO}_{2}\right)$ particles, and PSt particles. Next, the surface-initiated ATRP of hydrophilic monomers was conducted to generate a functional polymer brush layer. One-step hierarchically structured coatings of materials surface were also investigated.

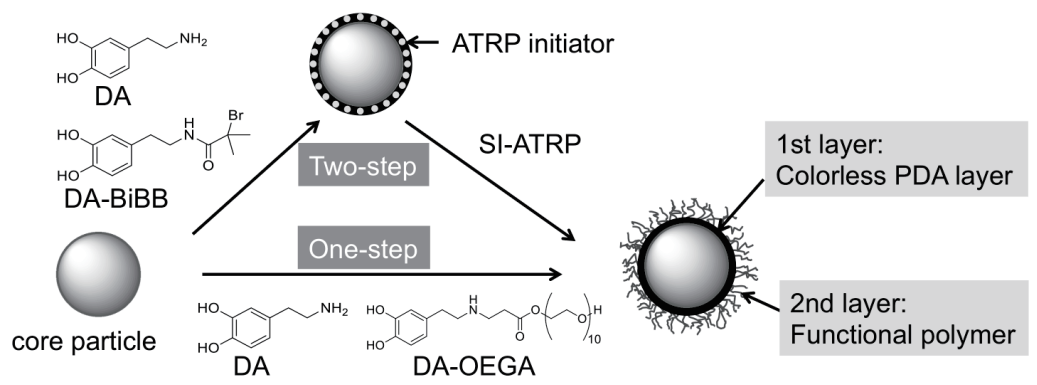

Fig. 1 Schematic representation of surface functionalization via hierarchically structured coatings using colorless PDA layer and polymer brush layer. 


\section{EXPERIMENTAL SECTION \\ 2.1 Materials}

Dopamine hydrochloride (DA), 2-bromoisobutyryl bromide (BiBB), tris(hydroxymethyl)aminomethane (Tris), styrene (St), copper(II) bromide $\left(\mathrm{CuBr}_{2}\right)$, sodium L-ascorbate, ethanol, triethylamine $\left(\mathrm{Et}_{3} \mathrm{~N}\right)$ $N, N$-dimethylformamide (DMF), and 2-hydroxyethyl methacrylate (HEMA) were purchased from Kanto Chemical. $\quad N$-isopropylacrylamide (NIPAM) was purchased from Wako Pure Chemical. $\mathrm{CaCO}_{3}$ particles (Vigot-15) were obtained from Shiraishi Kogyo Kaisha Ltd. $\mathrm{SiO}_{2}$ particles (MP-1040) were obtained from Nissan Chemical Ind. Ltd. Oligoethylene glycol methyl ether acrylate (OEGA: Blemmer AE-400, $\mathrm{n}=10$ ) was obtained from NOF Co. St was dried over calcium hydride and distilled under reduced pressure. NIPAM was recrystallized by hexane. HEMA was distilled under reduced pressure. Tris[2-(dimethylamino)ethyl]amine ( $\mathrm{Me}_{6}$ TREN) was synthesized according to methods reported in the literature [7]. All other chemicals and solvents were of reagent grade and used as received.

\subsection{Measurements}

Scanning electron microscopy (SEM) micrographs were obtained using a scanning electron microscope (JSM-6510A; JEOL) operated at 15-20 kV. The hydrodynamic diameter $\left(D_{\mathrm{h}}\right)$ of the particles in water was measured by dynamic light scattering (DLS) (ELSZ-1000ZS; Otsuka Electronics Co. Ltd). Infrared (IR) spectra were measured by an IR spectrophotometer (FTIR-420; JASCO).

\subsection{Two-step hierarchically structured coatings:} Preparation of polymer-grafted substrate@PDA/BiBB 2

DA (0.20 g, $1.06 \mathrm{mmol})$, BiBB (0.49 g, $2.12 \mathrm{mmol})$, and $\mathrm{Et}_{3} \mathrm{~N}(0.21 \mathrm{~g}, 2.12 \mathrm{mmol})$ in $\mathrm{DMF}(10 \mathrm{~mL})$ were stirred for $3 \mathrm{~h}$ at r.t. under $\mathrm{N}_{2}$. Core materials, Tris (1.21 $\mathrm{g}, 10 \mathrm{mmol})$, and deionized water $(90 \mathrm{~mL})$ were added to the mixture, and the mixture was stirred for $24 \mathrm{~h}$ at r.t. When $\mathrm{SiO}_{2}$ or PSt particles were used as a substrate, obtained particles were separated and purified repeatedly by centrifugation (14500 rpm for $20 \mathrm{~min}$ ) and redispersion. When glass plate, filter paper, or flexible PP film was used as a substrate, they were rinsed with water repeatedly.

Monomer (1.0 mmol), $\mathrm{CuBr}_{2}(6.7 \mathrm{mg}, 0.03 \mathrm{mmol})$, $\mathrm{Me}_{6}$ TREN (13.8 $\left.\mathrm{mg}, \quad 0.06 \mathrm{mmol}\right)$ and substrate@PDA/BiBB $\mathrm{Bi}_{2}$ dispersed in deionized water (10 $\mathrm{mL}$ ) were placed in a $20 \mathrm{~mL}$ glass tube. The mixture was deoxygenated by purging with $\mathrm{N}_{2}$ for 20 min and placed in a water bath at r.t. An $\mathrm{N}_{2}$-purged aqueous solution of sodium L-ascorbate $(11.9 \mathrm{mg}, 0.06 \mathrm{mmol}$ ) was added to the mixture. After $5 \mathrm{~h}$, the polymerization was stopped by purging with oxygen, and purification as described above.

\subsection{One-step hierarchically structured coatings:} Preparation of $\mathrm{CaCO}_{3} @ P D A / O E G A_{m}$ particles

DA (0.40 g, $2.11 \mathrm{mmol})$, OEGA (0-0.87 g, 0-1.69 $\mathrm{mmol})$, and $\mathrm{Et}_{3} \mathrm{~N}(147 \mu \mathrm{L}, 1.06 \mathrm{mmol})$ in DMF $(4 \mathrm{~mL})$ were stirred for $24 \mathrm{~h}$ at r.t. $\mathrm{CaCO}_{3}$ core particles $(10 \mathrm{mg})$, Tris $(0.12 \mathrm{~g}, 1.0 \mathrm{mmol})$, deionized water $(5 \mathrm{~mL})$, and ethanol $(4 \mathrm{~mL})$ were added to the mixture, and the mixture was stirred for $24 \mathrm{~h}$ at r.t. The obtained particles were separated and purified repeatedly by centrifugation (14500 rpm for $10 \mathrm{~min}$ ) and redispersion.

\section{RESULTS AND DISCUSSION}

\subsection{Two-step hierarchically structured coatings}

Several materials, including glass plate, filter paper, flexible PP film, $\mathrm{SiO}_{2}$ particles, and PSt particles, were coated with conventional PDA and $\mathrm{PDA} / \mathrm{BiBB}_{2}$ thin layer, respectively. As shown in Fig. 2a, the color of $\mathrm{PDA} / \mathrm{BiBB}_{0}$-coated materials was changed to dark brown: conventional PDA coating. In contrast, $\mathrm{PDA} / \mathrm{BiBB}_{2}$-coated materials were relatively colorless (Fig. 2b). When glass plate and flexible PP film were used as a substrate, $\mathrm{PDA} / \mathrm{BiBB}_{2}$-coated materials results in optically transparent and shows relatively same transparency with a bare glass plate and PP film, respectively (Fig. 3a). Furthermore, the wettability of PDA- or PDA/BiBB 2 -coated plates was measured by a static contact angle measurement. The contact angles for water were $33^{\circ}$ and $90^{\circ}$ on the hydrophilic glass plate surface and hydrophobic PP film, respectively (Fig. 3 bi,iv). After PDA-coating, the values were changed to $40^{\circ}$ and $43^{\circ}$, respectively (Fig. 3bii,v), indicating PDA was enough to generate films with uniform chemical structures independent of the substrate composition, in accordance with previous report [19]. For glass plate, the contact angles were relatively same on PDA- and $\mathrm{PDA} / \mathrm{BiBB}_{2}$-coated glass plate (Fig. 3bii,iii). On the other hand, the value on $\mathrm{PDA} / \mathrm{BiBB}_{2}$-coated PP film was enhanced to $66^{\circ}$ compared with PDA-coated PP film (Fig. 3bv,vi). This phenomenon is probably due to the thin thickness of the $\mathrm{PDA} / \mathrm{BiBB}_{2}$ layer thickness. The thicknesses of $\mathrm{PDA} / \mathrm{BiBB}_{2}$ on glass plate [17] and PSt particles [18], measured by atomic force microscopy (AFM) and SEM, were determined to be ca. $6 \mathrm{~nm}$ and 7 $\mathrm{nm}$, respectively, indicating thin thickness of $\mathrm{PDA} / \mathrm{BiBB}_{2}$ layer. When thickness of the PDA was thin, the wettability of PDA films depends on the surface (a)

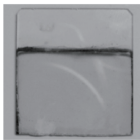

(b)

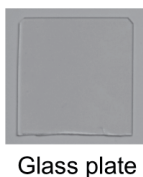

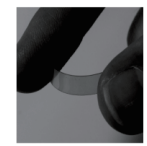

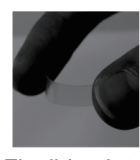

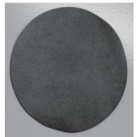

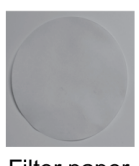

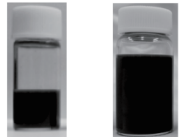

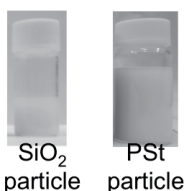

Fig. 2 Photographs of $\mathrm{PDA} / \mathrm{BiBB}_{\mathrm{n}}$ coated substrates with (a) $n=0$ and (b) $n=2$. (a)

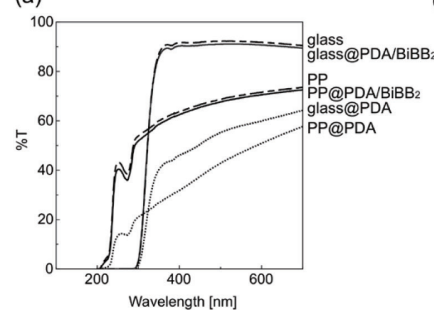

(b)

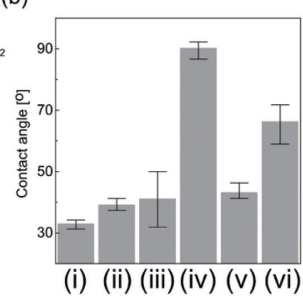

Fig. 3 (a) UV-vis spectra of plates. (b) Contact angle for water on (i) glass, (ii) glass@PDA, (iii) glass@PDA/BiBB ${ }_{2}$, (iv) PP, (v) PP@PDA, and (vi) $\mathrm{PP} @ \mathrm{PDA} / \mathrm{BiBB}_{2}$ 
property of substrates [19]. Thus, we believed that the contact angle on $\mathrm{PDA} / \mathrm{BiBB}_{2}$-coated $\mathrm{PP}$ film would be influenced by hydrophobic nature of PP.

The modification of $\mathrm{PDA} / \mathrm{BiBB}_{2}$ layer onto $\mathrm{SiO}_{2}$ particles was confirmed using IR spectroscopy (Fig. 4). Signals at $1665 \mathrm{~cm}^{-1}$ correspond to the $\mathrm{C}=\mathrm{O}$ vibration mode of a BiBB moiety was virtually disappeared since it was overlapped with the peak corresponds to $\mathrm{SiO}-\mathrm{H}$ stretching vibration signal (Fig. 4b). However, after SI-ATRP of 2-hydroxyethyl methacrylate (HEMA) onto $\mathrm{SiO}_{2} @ \mathrm{PDA} / \mathrm{BiBB}_{2}$ particles, major peaks at $1721 \mathrm{~cm}^{-1}$ $(\mathrm{C}=\mathrm{O}$ stretching vibration $)$ and at $1456 \mathrm{~cm}^{-1}(\mathrm{CO}-\mathrm{H}$ bending vibrations) appeared. No signal corresponding to PHEMA was obtained in the absence of PDA/BiBB layer. These results indicate the presence of ATRP initiating group and PHEMA brush layer in the $\mathrm{SiO}_{2} @ \mathrm{PDA} / \mathrm{BiBB}_{2}$ particles (Fig. 4c).

The PSt@PDA/BiBB 2 particles with $375 \mathrm{~nm}$ in diameter were used as a macroinitiator for SI-ATRP to construction of functional 2nd layers. Poly $(N$-isopropylacrylamide) (PNIPAM), one of the well-known thermoresponsive polymers, was selected as a grafted polymer. Fig. 5 shows the dependence of hydrodynamic diameter $\left(D_{\mathrm{h}}\right)$ on temperature measured by DLS for PNIPAM-grafted PSt@PDA/BiBB particles. The $D_{\mathrm{h}}$ of the particles decreased with the increase of temperature from 20 to $40{ }^{\circ} \mathrm{C}$, and the size distribution of the PNIPAM-grafted particles obtained from DLS keeps very narrow at each temperature. As shown in Fig. 5 inset, it can be seen that obtained particles exhibit similar values of the $D_{\mathrm{h}}$ at two temperatures even after several heating-cooling cycles, indicating there is no aggregation between particles during the thermal cycles.

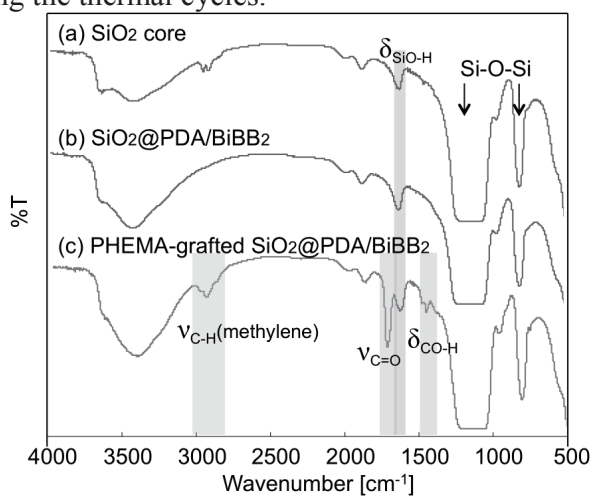

Fig. 4 IR spectra of (a) $\mathrm{SiO}_{2}$ core, (b) $\mathrm{SiO}_{2} @ \mathrm{PDA} / \mathrm{BiBB}_{2}$, (c) PHEMA-grafted $\mathrm{SiO}_{2} @ \mathrm{PDA} / \mathrm{BiBB}_{2}$ particles.

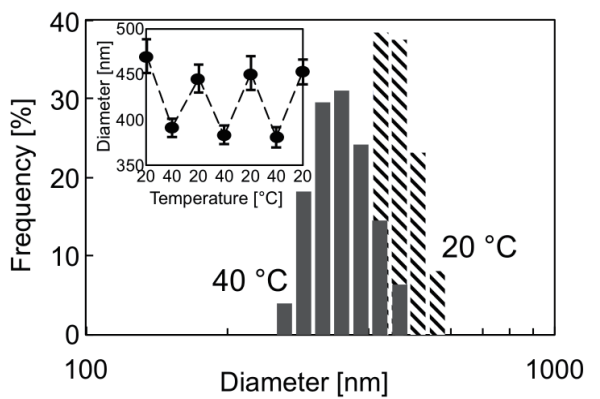

Fig. 5 Size distribution of PNIPAM-grated PSt@PDA/BiBB particles measured by DLS in water at $20^{\circ} \mathrm{C}$ and $40{ }^{\circ} \mathrm{C}$. Inset shows thermal-responsive swelling and shrinking cycles.

\subsection{One-step hierarchically structured coatings}

The above results indicated that the hierarchically structured coatings using colorless PDA thin layer and polymer brush layer is a practical technique for surface modification of materials. We further demonstrated the one-step hierarchically structured coatings. The PDA layer, which contains PEG moiety instead of BiBB, was directly coated on to material surfaces. Hydrophobic calcium carbonate $\left(\mathrm{CaCO}_{3}\right)$ particles, modified with fatty acids, were selected as a core material. While core $\mathrm{CaCO}_{3}$ particles did not dispersed in water (Fig. 6a), $\mathrm{CaCO}_{3} @ \mathrm{PDA} / \mathrm{OEGA}_{\mathrm{m}}$ particles were well dispersed. Fig. $6 b$ presents the photographs of the $\mathrm{CaCO}_{3} @ \mathrm{PDA} / \mathrm{OEGA}_{\mathrm{m}}$ particles with different feeds of OEGA/DA in water. The photo images of the PDA/OEGA $m(m=0-0.8)$-coated $\mathrm{CaCO}_{3}$ particles showed that their color changes decreased as the $\mathrm{m}$ value increased, in agreement with our previous report [17]. The dark brown coloration also decreased with increasing $\mathrm{Et}_{3} \mathrm{~N}$ concentration (Fig. 6c). In the preparation of OEGA/DA, the OEGA was reacted with the amino group of $\mathrm{DA}$ in the presence of $\mathrm{Et}_{3} \mathrm{~N}$ by Michael addition reaction. The formation of DA-OEGA prevented cyclization to the indole moiety, giving rise to the low cross-link densities and polymerization degree of the PDA. Thus, the color change of the PDA layer decreased depending on the $\mathrm{m}$ value or $\mathrm{Et}_{3} \mathrm{~N}$ concentration.

The modification of PDA/OEGA ${ }_{0.5}$ layer on $\mathrm{CaCO}_{3}$ core particles was confirmed by IR spectroscopy (Fig. 7) A rather sharp peak occurred at $1723 \mathrm{~cm}^{-1}$, which can be attributed to the $\mathrm{C}=\mathrm{O}$ stretching vibration mode of OEGA. The spectrum of the $\mathrm{CaCO}_{3} @ \mathrm{PDA} / \mathrm{OEGA}_{0.5}$ particles is similar to the combined spectrum of the $\mathrm{CaCO}_{3}$ core particles, OEGA, and PDA (Fig. 7c). These results strongly indicate that one-step hierarchically structured coatings via the in situ oxidative copolymerization of a DA and DA-OEGA solution onto $\mathrm{CaCO}_{3}$ surface were successfully demonstrated. To investigate the versatility of this method, we performed one-step hierarchically structured coatings on to $\mathrm{SiO}_{2}$ $\left(D_{\mathrm{h}}=130 \mathrm{~nm}\right)$ and PSt $\left(D_{\mathrm{h}}=190 \mathrm{~nm}\right)$ particles instead of $\mathrm{CaCO}_{3}$ particles (Fig. 6d). While $\mathrm{SiO}_{2} @ \mathrm{PDA} / \mathrm{OEGA} \mathrm{A}_{0.8}$ and PSt@PDA/OEGA 0.8 particles were well dispersed in water, the modified DA/OEGA ${ }_{0.8}$ layers are too thin for detection of signals using IR measurements. Further experiments are in progress to evaluate the effect of core materials on hierarchically structured coatings.

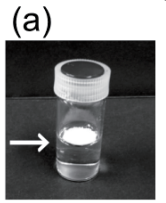

(c)

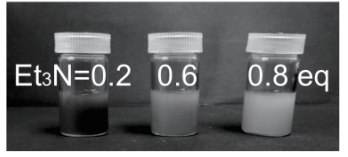

\section{(b)}

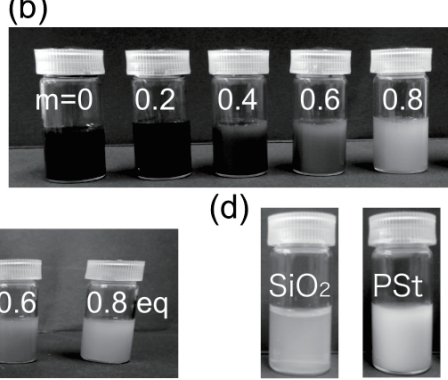

Fig. 6 Photographs of (a) $\mathrm{CaCO}_{3}$ core, (b) $\mathrm{CaCO}_{3} @ \mathrm{PDA} / \mathrm{OEGA}_{\mathrm{m}} \quad\left(\mathrm{Et}_{3} \mathrm{~N}=0.5 \mathrm{eq}\right), \quad(\mathrm{c})$ $\mathrm{CaCO}_{3} @ \mathrm{PDA} / \mathrm{OEGA}_{0.5}$, and (d) $\mathrm{SiO}_{2} @ \mathrm{PDA} / \mathrm{OEGA}_{0.8}$ and PSt@PDA/OEGA ${ }_{0.8}$ particles $\left(\mathrm{Et}_{3} \mathrm{~N}=0.8 \mathrm{eq}\right)$ in water. 


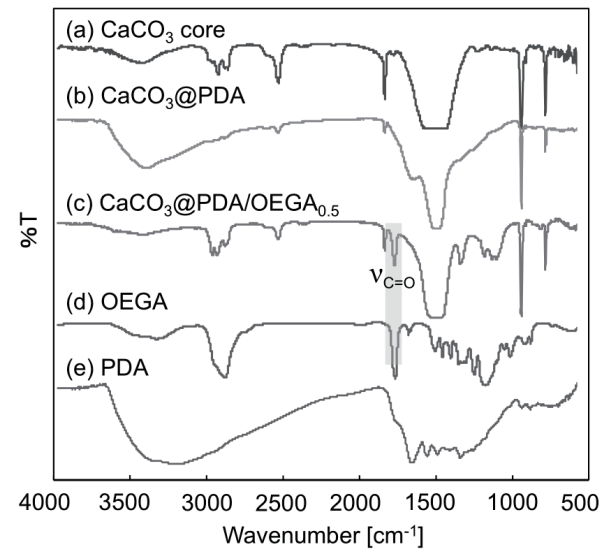

Fig. 7 IR spectra of (a) $\mathrm{CaCO}_{3}$ core particles, (b) $\mathrm{CaCO}_{3} @ \mathrm{PDA}$ particles, (c) $\mathrm{CaCO}_{3} @ \mathrm{PDA} / \mathrm{OEGA}_{0.5}$ particles, (d) OEGA, and (e) PDA.

\section{CONCLUSION}

In conclusion, hierarchically structured coatings onto material surfaces using colorless PDA thin layer and polymer brush layer were successfully demonstrated using following two procedures: (i) ATRP initiator-bearing colorless $\mathrm{PDA}$ layer $\left(\mathrm{PDA} / \mathrm{BiBB}_{2}\right.$ layer) has been prepared onto various types substrates, and then the surface-initiated ATRP was conducted to generate a functional polymer brush layer (two-step hierarchically structured coatings). Surface functionality will be controlled by select monomers for SI-ATRP. (ii) PEG polymer chain-containing colorless PDA layer $\left(\mathrm{PDA} / \mathrm{OEGA}_{0.5}\right.$ ) was directly covered onto $\mathrm{CaCO}_{3}$ particles (one-step hierarchically structured coatings). One-step methodology will be applied to a broad range of functional compounds other than PEG polymer to give functional materials. This work provides a simple, general, and versatile method for the surface functionalization of materials, since PDA can be used to cover a variety of material surfaces.

Acknowledgements: The authors thank Shiraishi Kogyo Kaisha Ltd., Nissan Chemical Ind. Ltd., and NOF Co. for providing $\mathrm{CaCO}_{3}$ particles (Vigot-15), $\mathrm{SiO}_{2}$ particles (MP-1040), and OEGA (Blemmer AE-400), respectively.

\section{REFERENCES}

[1] X. Y. Huang and M. J. Wirth, Anal. Chem., 69, 4577 (1997).

[2] M. Ejaz, K. Ohno, Y. Tsujii, and T. Fukuda, Macromolecules, 33, 2870 (2000).

[3] K. Min, J. Hu, C. Wang, and A. Elaïssari, J. Polym. Sci. A Polym. Chem., 40, 892 (2002).

[4] S. Edmondson, V. L. Osborne, and W. T. S. Huck, Chem. Soc. Rev., 33, 14 (2004).

[5] R. Barbey, L. Lavanant, D. Paripovic, N. Schüwer, C. Sugnaux, S. Tugulu, and H. A. Klok, Chem. Rev., 109, 5437 (2009).

[6] F. J. Xu, K. G. Neoh, and E. T. Kang, Prog. Polym. Sci., 34, 719 (2009).

[7] M. Kohri, M. Sato, F. Abo, T. Inada, M. Kasuya, T. Taniguchi, and T. Nakahira, Eur. Polym. J., 47, 2351 (2011).
[8] W. Huang, J. B. Kim, M. L. Bruening, and G. L. Baker, Macromolecules, 35, 1175 (2002)

[9] K. Ohno, T. Morinaga, K. Koh, Y. Tsujii, and T. Fukuda, Macromolecules, 38, 2137 (2005).

[10] W. Ma, H. Otsuka, and A. Takahara, Chem. Commun., 47, 5813 (2011).

[11] H. Lee, S. M. Dellatore, W. M. Miller, and P. B. Messersmith, Science, 318, 426 (2007).

[12] J. H. Waite, Nat. Mater., 7, 8 (2008).

[13] H. Lee, J. Rho, and P. B. Messersmith, Adv. Mater., 21, 431 (2009).

[14] J. Ryu, S. H. Ku, H. Lee, and C. B. Park, $A d v$. Funct. Mater., 20, 2132 (2010).

[15] Q. Ye, F. Zhou, and W. Liu, Chem. Soc. Rev., 40, 4244 (2011).

[16] M. Kohri, Y. Nannichi, H. Kohma, D. Abe, T. Kojima, T. Taniguchi, and K. Kishikawa, Colloids Surf. A: Physicochem. Eng. Aspects, 449, 114 (2014).

[17] M. Kohri, Y. Shinoda, H. Kohma, Y. Nannichi, M. Yamauchi, S. Yagai, T. Kojima, T. Taniguchi, and K. Kishikawa, Macromol. Rapid Commun., 34, 1220 (2013).

[18] M. Kohri, H. Kohma, Y. Shinoda, M. Yamauchi, S. Yagai, T. Kojima, T. Taniguchi, and K. Kishikawa, Polym. Chem., 4, 2696 (2013).

[19] K. Kang, I. S. Choi, and Y. Nam, Biomaterials, 32, 6374 (2011).

(Received February 24, 2014; Accepted March 28, 2014) 